\title{
Comunicación
}

\section{Enanismo hipofisario en un canino Poodle: hallazgos clínicos y de laboratorio}

\author{
Pituitary dwarfism in a Poodle dog: clinical and laboratory findings \\ Kariny S. Cruz ${ }^{1}$, Daniela S. Wondracek ${ }^{1}$, Cristiane M.F. Melo ${ }^{1,2}$
}

\section{Resumien}

\begin{abstract}
El presente trabajo tuvo como objetivo evaluar los hallazgos clínicos y de laboratorio de un caso de enanismo hipofisario en un canino de raza Poodle, hembra de 4 meses. Los signos clínicos observados fueron un menor tamaño corporal, pelaje suave y lanoso, retención del sub-pelaje, ausencia de pelos primarios, descamación de piel de la región de la cabeza y dientes de leche. Los análisis hormonales indicaron valores de $0.5 \mathrm{ng} / \mathrm{ml}$ para la hormona del crecimiento $(\mathrm{GH}), 0.44 \mathrm{ng} / \mathrm{ml}$ para $\mathrm{T} 3$ total y $1.44 \mathrm{ng} / \mathrm{dl}$ para T4 libre. Los niveles de GH estaban debajo de lo normal, indicando así, un cuadro de enanismo hipofisario.
\end{abstract}

Palabras clave: hipotálamo, hipófisis, somatotropina, tiroides

\section{Abstract}

This paper aimed to evaluate the clinical and laboratory findings of a case of pituitary dwarfism in a 4-month-old female Poodle dog. The clinical signs observed were a reduced body size, soft and woolly coat, retention of the sub-coat, absence of primary hairs, scaling of the skin of the head region and deciduous teeth. Hormonal analyses indicated values of $0.5 \mathrm{ng} / \mathrm{ml}$ for growth hormone $(\mathrm{GH}), 0.44 \mathrm{ng} / \mathrm{ml}$ for total $\mathrm{T} 3$ and $1.44 \mathrm{ng} / \mathrm{dl}$ for free T4. GH levels were below normal, thus indicating a pituitary dwarfism.

Key words: hypothalamus, hypophysis, somatotropin, thyroid

\footnotetext{
${ }^{1}$ Faculdade de Ciências Exatas e Agrárias, Centro Universitário da Grande Dourados (UNIGRAN), Mato Grosso do Sul, Brasil

${ }^{2}$ E-mail: crisalicemelo@gmail.com
}

Recibido: 9 de abril de 2019

Aceptado para publicación: 18 de enero de 2020

Publicado: 31 de marzo de 2020 


\section{INTRODUCCIÓN}

El enanismo hipofisario canino es causado por una deficiencia congénita de la hormona del crecimiento, asociado a una distención cística congénita o persistencia de la porción intraselar del ducto cráneofaríngeo (fosa de Rathke) (Nelson y Couto, 1998). En este caso, el ectodermo orofaríngeo de la bolsa de Rathke es insuficiente para diferenciar las células secretoras de hormonas tróficas de la pars distalis, que resulta en la formación de un quiste multilobulado, que aumenta progresivamente el volumen en la silla turca $(\mathrm{Ca}-$ pen, 1998).

Quistes grandes pueden causar la compresión y consecuente atrofia de las células hipofisarias. La deficiencia de las hormonas hipofisarias favorece la formación de quistes e hipoplasia pituitaria, con ausencia parcial o total de la hipófisis (Nelson y Couto 1998; Kooistra et al., 2000; Santos y Alessi, 2010).

Kooistra et al. (2000) indican que las principales características físicas exhibidas en el enanismo son el retardo del crecimiento y el desarrollo interrumpido del pelo. El tamaño de los animales varía de acuerdo con la falla en la formación de la adenohipófisis (Capen, 1998). Los animales afectados generalmente presentan tamaños normales al nacer y hasta el segundo mes de vida, para posteriormente presentar una tasa de crecimiento más lenta. Al tercer o cuarto mes de edad, los pacientes con el síndrome de enanismo son obviamente perceptibles dentro de la camada (Santos y Alessi, 2010). Otros signos clínicos que se pueden observar son: atraso mental, retención de pelaje secundario (borra), alopecia simétrica bilateral, híperpigmentación cutánea, descamación, persistencia de dientes caninos deciduos, vocalización de cachorro, criptorquidismo en el macho y anestro persistente en las hembras (Gaspar y Amaral, 1995; Benchekroun y Rosenberg, 2011).
En los exámenes complementarios se puede observar hipofosfatemia, hipoalbuminemia, anemia y azotemia moderada como consecuencia de la diminución de los procesos anabólicos resultante de la deficiencia de la hormona de crecimiento - GH (Nelson y Couto, 1998). Existen evidencias de hipotiroidismo secundario debido a la posible asociación de deficiencias hormonales combinadas, donde los niveles de tiroxina (T4) circulante presenta valores séricos bajos (Kooistra, 2009)

Los animales con enanismo hipofisiario desarrollan sin problemas adicionales hasta los 2-3 años, cuando se inician los signos sistémicos (Benchekroun y Rosenberg, 2011). El tratamiento precoz del enanismo hipofisario puede ser realizado tanto con administración de $\mathrm{GH}$ o de fármacos alfa2-adrenérgicos (xilazina). La comercialización de GH canino no está disponible para uso terapéutico, habiendo en el mercado GH de cerdo y humano, de modo que la formación de anticuerpos contra GH humano puede impedir su uso en la terapéutica canina (Kooistra, 2009).

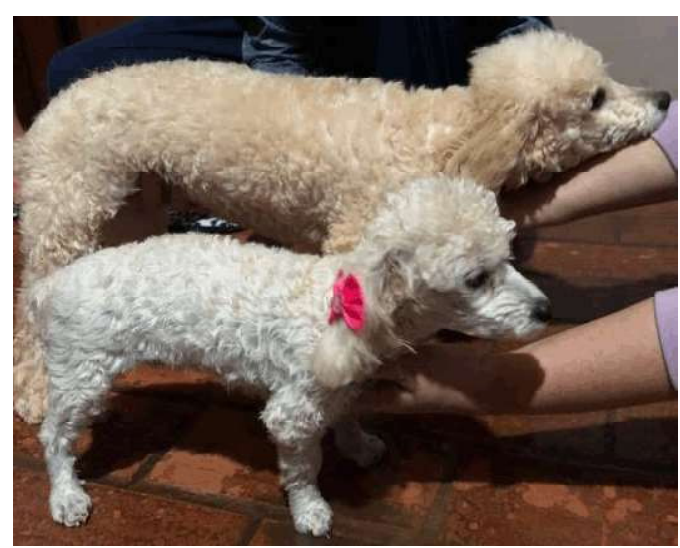

Figura 1. Paciente canino de raza Poodle diagnosticado con enanismo hipofisario en la Clínica Veterinaria de la Centro Universitario de la Grande Dourados (INIGRAN), 2018. Fuente: Kariny Sanches Cruz 
Cuadro 1. Hemograma de un canino Poodle con enanismo hipofisario atendido en la Clínica Veterinaria de la UNIGRAN en Dourados, MS, Brasil (2018)

\begin{tabular}{lcc}
\hline & Valores & Valores de referencia $^{1}$ \\
\hline Eritrograma & 4.9 & $\left(5,5-8,5 \times 10^{6}\right)$ \\
Eritrocitos $\left(\mu \mathrm{l} \times 10^{6}\right)$ & 10.4 & $(12-18)$ \\
Hemoglobina $(\mathrm{g} / \mathrm{dl})$ & 31.4 & $(37-55)$ \\
Hematocrito $(\%)$ & 64.0 & $(60-77)$ \\
VGM $(\mathrm{fl})$ & 33.1 & $(35-42)$ \\
CHCM $(\%)$ & 195.000 & $\left(200-500 \times 10^{3}\right)$ \\
Plaquetas $(\mu \mathrm{l})$ & & \\
Leucograma & 7800 & $\left(60-170 \times 10^{3}\right)$ \\
Leucocitos $(\mu \mathrm{l})$ & 0 & $(0)$ \\
Mielocitos & 0 & $0(0-300)$ \\
Metamielocitos & 0 & $(1,000-11,500)$ \\
Leuc. En banda & 32 & $(150-1,350)$ \\
Leuc. Segmentados & 62 & $(100-1,350)$ \\
Linfocitos & 5 & raros \\
Monocitos & 1 & 0 \\
Eosinofilos & 0 & \\
Basófilos & &
\end{tabular}

VCM: Volumen Corpuscular Medio, CHCM: Concentración de Hemoglobina Corpuscular Media

${ }^{1}$ Laboratorio de Patología Clínica Veterinária, Unigran, Dourados (2018) (Thrall et al., 2015)

Se reporta predisposición racial, principalmente en caninos Pastor Alemán; sin embargo, también se ha observado en caninos Weimaraner, Spitz, Pinscher Toy y Carnelian Bear (Gaspar y Amaral, 1995; Nelson y Couto, 1998; Benchekroun y Rosenberg, 2011); no existiendo predisposiciones comprobadas con relación al sexo (Muller et al., 1985).

\section{Caso Clínico}

Se presenta un caso de enanismo hipofisario en un paciente Poodle, atendido en la Clínica Veterinaria del Centro Universitario de la Grande Dourados (UNIGRAN) en la ciudad de Dourados, Estado de Mato Grosso do Sul (MS), Brasil.
Cuadro 2. Valores bioquímicos séricos de un canino Poodle con enanismo hipofisario. Clínica Veterinaria, UNIGRAN (Brasil. 2018)

\begin{tabular}{lcc}
\hline & Resultado & $\begin{array}{c}\text { Valores de } \\
\text { referencia }^{1}\end{array}$ \\
\hline $\begin{array}{l}\text { Alanina } \\
\text { aminotransferasa } \\
\text { (UI/l) }\end{array}$ & 34 & $21-73$ \\
$\begin{array}{l}\text { Fosfatasa } \\
\text { alcalina (UI/l) }\end{array}$ & 171 & $20-156$ \\
$\begin{array}{l}\text { Creatinina } \\
\text { (mg/dl) }\end{array}$ & 0.9 & $0.5-1.5$ \\
$\begin{array}{l}\text { Urea (mg/dl) } \\
{ }^{1} \text { Laboratorio de Patología Clínica Veteri-nária, } \\
\text { Unigran, Dourados (2018), (Thrall et al., 2015) }\end{array}$
\end{tabular}


Cuadro 3. Análisis hormonal de un canino Poodle con enanismo hipofisario atendido en la Clínica Veterinaria de la UNIGRAN en Dourados, MS, Brasil (2018)

\begin{tabular}{lcc}
\hline Hormona & Resultado & Valores de referencia $^{1}$ \\
\hline Hormona de crecimiento $(\mathrm{GH}), \mathrm{ng} / \mathrm{ml}$ & 0.50 & $1.8-6.4$ \\
T3 total, ng/ml & 0.44 & $0.30-1.50$ \\
T4 libre, $\mathrm{ng} / \mathrm{dl}$ & 1.44 & $0.70-1.80$ \\
\hline
\end{tabular}

T3: Triyodotiroxina; T4: Tiroxina

${ }^{1}$ Centro de Diagnóstico (Diagnóstika), Dourados, Brasil, T3 yT4 (Nelson y Couto, 1998)

El canino era hembra, de 4 meses y 400 $\mathrm{g}$ de peso y fue llevado a consulta por presentar señales de crecimiento tardío con relación a los hermanos de la camada (Figura 1). La propietaria indica que el paciente descendía de animales con estaturas normales.

En el examen clínico, los signos observados, además del tamaño reducido, fuero pelaje suave y lanoso, retención de subpelaje, ausencia de pelos primarios, descamación de piel en la región de la cabeza y dientes de leche.

Se colectó una muestra de sangre en un tubo de ensayo conteniendo EDTA, para un hemograma completo. El análisis se hizo en un contador automático de células (HORIBA Analizador, modelo ABS Micros ESV 60). El conteo diferencial de leucocitos, para determinar el porcentaje de basófilos, eosinófilos y neutrófilos en diferentes etapas de maduración, así como de linfocitos y monocitos fue realizado mediante microscopía óptica (X1000) a partir de frotis sanguíneos teñidos con Panóptico Rápido $\left(\right.$ Interlab $\left.^{\circledR}\right)$.

Se realizaron análisis bioquímicos utilizando un conjunto de reactivos comerciales (Gold Analiza Diagnóstica) para cuantificar las concentraciones de urea, creatinina, y las actividades de las enzimas alanina aminotransferasa (ALT) y fosfatasa alcalina (FA) en un espectrofotómetro semiautomático
(Bioplus 2000 ${ }^{\circledR}$ ). Los análisis hematológicos y bioquímicos sanguíneos fueron realizados en el Laboratorio de Patología Clínica Veterinaria de la UNIGRAN.

El animal fue derivado a un centro de diagnóstico (Diagnóstika), en la misma ciudad, para exámenes hormonales complementarios: hormona del crecimiento $(\mathrm{GH})$, triyodotironina ( $\mathrm{T} 3$ total) y tiroxina (T4 libre) mediante la técnica de inmunoensayo de fluorescencia.

\section{Resultados de Laboratorio}

En el hemograma se observó un cuadro de normocitosis e hipocromía. En la serie blanca se encontró un cuadro de neutropenia y linfocitosis. Además, hubo un cuadro de trombocitopenia (Cuadro 1). Los resultados del análisis bioquímico se muestran en el Cuadro 2 y de los análisis hormonales en el Cuadro 3 .

\section{Discusión}

En análisis hormonales de enanismo hipofisario en caninos Pastor Alemán se han reportado valores de $\mathrm{GH}$ por debajo del nivel normal (0.03 ng/ml) (Gaspar y Amaral, 1995; Júnior et al., 2016), lo cual corrobora los hallazgos del presente trabajo. 
Laporte (2012) resalta que se debe prestar atención a las alteraciones de crecimiento y lesiones dermatológicas sugestivas del cuadro de enanismo hipofisario con el fin de realizar un diagnóstico precoz, ya que este síndrome se puede manifestar en cualquier raza. Así, Noda et al. (1983) observó en un perro Pastor Alemán áreas alopécicas simétricas con hiperpigmentación en las extremidades.

La anemia observada en el animal del presente estudio puede deberse a la disminución de la función renal (Kooistra et al., 2000), la cual ocasiona una menor síntesis de eritropoyetina, citocina glucoproteica que estimula la producción de eritrocitos por la medula ósea. Por otro lado, los niveles aumentados de fosfatasa alcalina son frecuentes de encontrar en animales jóvenes, pues la actividad osteoblástica es intensa (Thrall, 2015). En este estudio, el animal presentó anemia normocítica hipocrómica, tal y como fue encontrado por Kooistra et al. (2000).

Los niveles de las hormonas tiroideas no estaban afectados, posiblemente debido a que el animal se encontraba en crecimiento y no había manifestado los primeros signos sistémicos que aparecen en el animal adulto (Benchekroun y Rosenberg, 2011).

\section{Literatura Citada}

1. Benchekroun G, Rosenberg D. 2011. Endocrinopatias atípicas caninas e felinas. Veterinary Focus 21: 37-38.

2. Capen H. 1998. Sistema endocrino. Em: Carlton WW, McGavin MD (eds). Patologia veterinária especial de Thomson. $2^{\circ}$ ed. Porto Alegre: ArtMed. p 266-304.
3. Gaspar LF, Amaral AS. 1995. Nanismo hipofisário em um canino: achados clínicos e laboratoriais. Ciência Rural 25: 465-468. doi: 10.1590/S010384781995000300024

4. Júnior HLS, Andrade JV, Mello ANR, Almeida MAS, Sousa FV. 2016. Aspectos clínicos e patológicos do nanismo hipofisário em cão. Rev Múltipla Brasília 30: 137-141.

5. Kooistra HS, Voorhout G, Mol JA, Rijnberk A. 2000. Combined pituitary hormone deficiency in German Shepherd dog with dwarfism. Domest Anim Endocrinol 19: 177-190. doi: 10.1016/ s0739-7240(00)00074-6

6. Kooistra HS. 2009. Nanismo hipofisário. En: Mooney CT, Peterson ME (eds). Manual de endocrinologia canina e felina. $3^{\circ}$ ed. São Paulo: Rocca. p 217-223.

7. Laporte SM. 2012. Nanismo hipofisário canino: Revisão de literatura e relato de caso. Belo Horizonte, Brasil: Univ. Federal de Minas Gerais. 21 p.

8. Muller GH, Kirk RW, Scott DW. 1985. Dermatologia dos pequenos animais. $3^{\circ}$ ed. São Paulo: Manole. 1130 p.

9. Nelson RW, Couto CG 1998. Distúrbios do hipotálamo e da hipófise. En: Medicina interna de pequenos animais. 2 . ed. Rio de Janeiro: Guanabara Koogan. p 547-549.

10. Noda RS, Hagiwara MK, Iwasaki M. 1983. Panhipopituitarismo juvenil em cão. Rev Fac Med Vet Zootec Univ S Paulo 20: 155-159. doi: 10.11606/ issn.2318-3659.v20i2p155-159

11. Santos RL, Alessi AC. 2010. Patologia do sistema endócrino. En: Patologia veterinária. São Paulo: Roca. p. 783-784.

12. Thrall MA. 2015. Hematologia e bioquímica clínica veterinária. $2^{\circ}$ ed. São Paulo: Roca. 678 p. 